\title{
Ulcerative Colitis with Positivity for Proteinase 3-Antineutrophil Cytoplasmic Antibody
}

\author{
Keiko Suzuki Satoshi Nakao Akihiro Suzuki Masahiko Inamori Hiroshi lida \\ Hiroki Endo Tomoyuki Akiyama Kyoko Yoneda Koji Fujita Hirokazu Takahashi \\ Masato Yoneda Yasunobu Abe Noritoshi Kobayashi Kensuke Kubota \\ Satoru Saito Atsushi Nakajima \\ Gastroenterology Division, Yokohama City University School of Medicine, Yokohama, Japan
}

Dear Sir,

A 33-year-old woman was admitted to our hospital with cough and fever (over $39.0^{\circ} \mathrm{C}$ ). About 1 month prior to the current admission, she had presented with fever (over $39.0^{\circ} \mathrm{C}$ ), diarrhea and bloody bowel discharge. On admission, her hemoglobin was $8.9 \mathrm{~g} / \mathrm{dl}$ and the serum C-reactive protein level was $6.8 \mathrm{mg} / \mathrm{dl}$. Colonoscopy revealed flare and erosions in the entire large intestine and the patient was diagnosed as having ulcerative colitis (fig. 1). Myeloperoxidase-antineutrophil cytoplasmic antibody (MPO-ANCA) was negative, whereas the proteinase 3-antineutrophil cytoplasmic antibody (PR3ANCA) titer was increased to 135 EU. As the patient was PR3-ANCA-positive, we focused our examination on the lungs, kidneys and nasopharynx, which are the most frequent sites of involvement in Wegner's granulomatosis. However, these examinations revealed no specific findings. With mesalazine therapy and bowel rest for 10 days, the patient became afebrile, her cough remitted and the gastrointestinal symptoms, namely, diarrhea and bloody bowel discharge, resolved.

ANCA is an autoantibody directed against the constituents of neutrophil granulocytes [1]. There are two basic ANCA patterns: the cytoplasmic (C-
ANCA) and the perinuclear (P-ANCA). CANCA appears as a granular, diffuse cytoplasmic fluorescence, often with accentuated fluorescence around the nuclear lobes, and typical P-ANCA reactivity is observed as a homogeneous rim-like staining of the perinuclear cytoplasm [2]. The antigen recognized by C-ANCA has been proven to be proteinase 3 (PR3), and the first antigen recognized by P-ANCA was

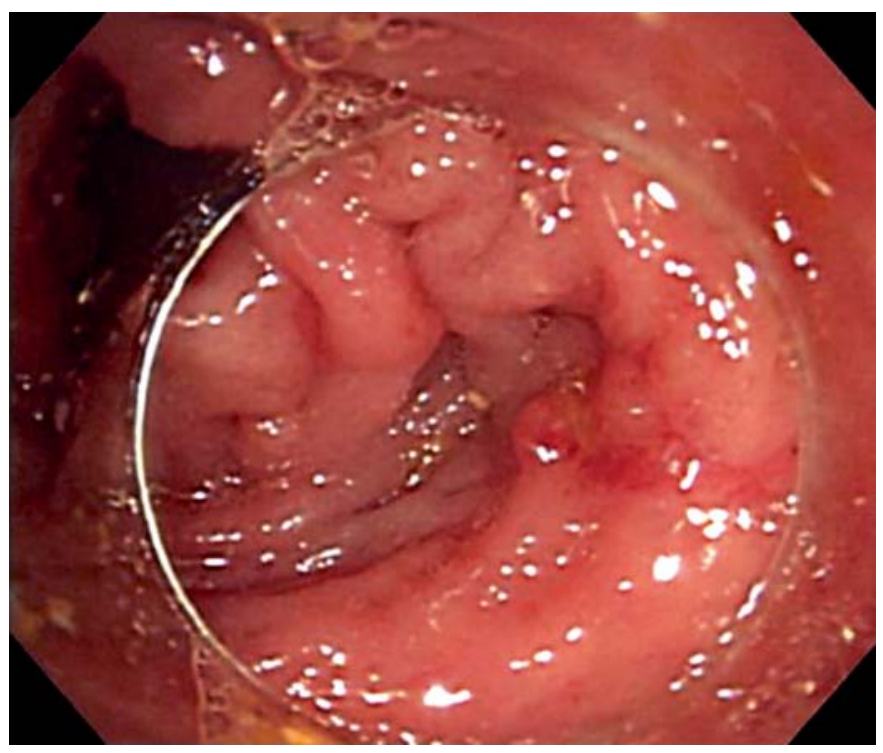

Fig. 1. Colonoscopy revealed erosions in the sigmoid colon that were compatible with ulcerative colitis.

\section{KARGER}

Fax +41613061234 E-Mail karger@karger.ch www.karger.com
(C) 2008 S. Karger AG, Base 0012-2823/08/0774-0157\$24.50/0

Accessible online at: www.karger.com/dig
Masahiko Inamori

Yokohama City University, School of Medicine, Gastroenterology Division

3-9 Fukuura, Kanazawa-ku, Yokohama, Kanagawa 236-0004 (Japan)

Tel. +81 45787 2640, Fax +81 457843546

E-Mail inamorim@med.yokohama-cu.ac.jp 
identified as myeloperoxidase (MPO) [1]. Antibodies to PR3 are highly specific for the diagnosis of Wegner's granulomatosis, and antibodies to MPO are associated with microscopic polyangiitis, Churg-Strauss syndrome, or idiopathic necrotizing crescentic glomerulonephritis [1]. Although PANCA is often seen in ulcerative colitis patients and its usefulness as a serological marker in IBD has been discussed in some papers, few PR3-ANCA-positive cases of ulcerative colitis have been reported.

In conclusion, we have reported a patient with PR3-ANCA-positive ulcerative colitis who was successfully treated with mesalazine. Further studies are needed to determine the relationship between ulcerative colitis and PR3-ANCA.

\section{References}

1 Roozendaal C, Kallenberg CG: Are anti-neutrophil cytoplasmic antibodies (ANCA) clinically useful in inflammatory bowel disease (IBD)? Clin Exp Immunol 1999;116: 206-213.

2 Papp M, Norman GL, Altorjay I, Lakatos PL: Utility of serological markers in inflammatory bowel diseases: gadget or magic? World J Gastroenterol 2007;13:2028-2036. 